\title{
Efficient Broadcasting in Ad Hoc Wireless Networks Using Directional Antennas
}

\author{
Fei Dai, Member, IEEE, and Jie Wu, Senior Member, IEEE
}

\begin{abstract}
Using directional antennas to conserve bandwidth and energy consumption in ad hoc wireless networks (or simply ad hoc networks) is becoming popular in recent years. However, applications of directional antennas for broadcasting have been limited. We propose a novel broadcast protocol called directional self-pruning (DSP) for ad hoc wireless networks using directional antennas. DSP is a nontrivial generalization of an existing localized deterministic broadcast protocol using omnidirectional antennas. Compared with its omnidirectional predecessor, DSP uses about the same number of forward nodes to relay the broadcast packet, while the number of forward directions that each forward node uses in transmission is significantly reduced. With the lower broadcast redundancy, DSP is more bandwidth and energy-efficient. DSP is based on 2-hop neighborhood information and does not rely on location or angle-ofarrival (AoA) information. Two special cases of DSP are discussed: the first one preserves shortest paths in reactive routing discoveries; the second one uses the directional reception mode to minimize broadcast redundancy. DSP is a localized protocol. Its expected number of forward nodes is $O(1)$ times the optimal value. An extensive simulation study using both custom and $n s 2$ simulators shows that DSP significantly outperforms both omnidirectional broadcast protocols and existing directional broadcast protocols.
\end{abstract}

Index Terms-Ad hoc wireless networks, broadcasting, directional antennas, localized algorithms, self-pruning, simulation.

\section{INTRODUCTION}

$\mathrm{U}$ SING smart antennas (i.e., directional antennas) to conserve bandwidth and energy consumption in wireless communications is becoming popular in recent years [12], [22]. Compared with the omnidirectional antennas, a smart antenna can form directional beams for both transmission and reception, which achieves better signal-to-noise ratio (SNR) and reduces interference. Benefits of directional antennas include capacity and range increases, supporting new services such as location estimation, better security, and reduced multipath propagation [12]. Many network protocols have been proposed for using directional antennas in ad hoc networks [5], [11], [17], [27]. However, most of them focused on the MAC layer, and research on the application of directional antennas in unicasting and broadcasting has been limited.

Broadcasting is frequently used in ad hoc networks not only for data dissemination, but also for route discovery in reactive unicast routing protocols. Blind flooding has been the most popular form of broadcasting because of its simplicity. In blind flooding, every node forwards the broadcast packet exactly once. The major drawback of blind flooding is its high cost and excessive redundancy, which causes the broadcast storm problem [28]. Both probabilistic

- F. Dai is with the Department of Electrical and Computer Engineering, North Dakota State University, Fargo, ND 58105.

E-mail: fdai@ece.ndsu.edu.

- J. Wu is with the Department of Computer Science and Engineering, Florida Atlantic University, Boca Raton, FL 33431.

E-mail: jie@cse.fau.edu.

Manuscript received 15 Feb. 2005; revised 13 May 2005; accepted 9 June 2005; published online 24 Feb. 2005.

Recommended for acceptance by I. Stojmenovic, S. Olariu, and

D. Simplot-Ryl.

For information on obtaining reprints of this article, please send e-mail to: tpds@computer.org, and reference IEEECS Log Number TPDSSI-0126-0205.
[3], [28] and deterministic [14], [15], [20], [21], [26] approaches have been proposed for efficient broadcasting in ad hoc networks. Probabilistic approaches use no [28] or limited [3] neighborhood information and require relatively high broadcast redundancy to maintain an acceptable delivery ratio. Deterministic approaches select a few forward nodes based on topology information to achieve full delivery. Most deterministic broadcast schemes in ad hoc networks are localized. A localized algorithm determines the status of each node (forward or nonforward) based on its $k$-hop neighborhood information, where $k$ is a small constant. Localized deterministic algorithms are more efficient than probabilistic approaches [30]. Nevertheless their efficiency can be further improved. Wireless nodes with directional antenna can control their radiation pattern to reduce broadcast redundancy. Several protocols [4], [10], [9], [25] have been proposed for efficient broadcasting using directional antennas. However, most of them are probabilistic approaches, depend on location or angle-of-arrival (AoA) information, or assume specific antenna models. None of them uses the directional reception mode.

In this paper, we propose a novel broadcast protocol called directional self-pruning (DSP), which extends a localized deterministic broadcast protocol (called selfpruning) for ad hoc networks with omnidirectional antennas [31]. Extending the omnidirectional self-pruning scheme to use directional antennas is nontrivial. We show that the original self-pruning algorithm in [31] must be enhanced carefully to avoid broadcast failure without being overly conservative. In certain occasions, it takes more forward nodes in DSP than in omnidirectional self-pruning to guarantee full delivery. Compared with its omnidirectional predecessor, DSP minimizes the interference and energy consumption by switching off transmission in 
unnecessary directions. Our simulation results show that, by partitioning the transmission range of each node into four directions, DSP uses about 10 percent more forward nodes than the original self-pruning, while reducing the transmission cost by 45 percent. We also implemented DSP on $n s 2$ and showed that it outperforms existing localized directional broadcast schemes in terms of efficiency and/or reliability.

DSP does not rely on location or AoA information. In DSP, each node is equipped with only 2-hop neighborhood information (or simply 2-hop information), which is collected via two rounds of "Hello" message exchanges among neighbors. The direction information (i.e., how to form a directional beam to reach a specific neighbor) is included in the 2-hop information and does not cause extra overhead to collect. DSP uses a general antenna model with fewer assumptions than existing models and, therefore, adapts well to a wide range of antenna techniques. In the new antenna model, directional beams can be irregular, overlapping, and unaligned. We also introduce two variations of DSP that support shortest path routing and directional reception mode.

The remainder of this paper is organized as follows: Section 2 reviews existing broadcast schemes using omnidirectional or directional antennas. Section 3 defines the efficient broadcasting problem and introduces the omnidirectional self-pruning scheme. We also provide our antenna model and neighborhood discovery scheme in this section. Section 4 describes the DSP algorithm and its properties. Section 5 discusses two variations of the DSP supporting shortest path routing and the directional reception mode, respectively. Simulation results are presented in Section 5, and Section 6 concludes this paper.

\section{Related Work}

Many deterministic broadcast schemes have been proposed for ad hoc networks using omnidirectional antennas. A deterministic broadcast algorithm is equivalent to an algorithm that forms a connected dominating set (CDS). The problem of finding a minimal CDS was proven NPcomplete. Approximation algorithms exist, but are either centralized [7], cluster-based [1], or location-based [13]. Centralized and cluster-based algorithms have slow convergency in mobile networks. Location-based algorithms rely on external devices such as GPS receivers, which cause extra cost. Localized broadcast algorithms can be further divided into neighbor designating algorithms and self-pruning algorithms. In neighbor-designating [14], [15], [21], each forward node selects a few 1-hop neighbors as new forward nodes to cover its 2-hop neighbors. In self-pruning [20], [26], [6], each node determines it own status (forward or nonforward). A generic self-pruning scheme was proposed in [31].

Application of directional antennas in localized broadcasting is limited in literature. Most of them are probabilistic approaches [4], [9], [25]. Choudhury and Vaidya [4] proposed to reduce the broadcast redundancy in relaying routing request by switching off transmissions in directions toward the last forward node. Hu et al. [9] presented three schemes to improve the broadcast efficiency. In the first scheme, each node switches off its transmission beams toward known forward nodes. In the second and third schemes, each forward node designates only one neighbor as a forward node in each direction. In the third scheme, the selection of forward nodes is aided by location information. Shen et al. [25] devised directional versions of probabilistic protocols in [28]. Only two localized deterministic schemes were proposed [10], [25]. Lim and Kim's neighbor elimination [14] was extended in [25], where each node switches off transmission in a direction, if all neighbors in this direction are also neighbors of a known forward node. In [10], each node forms a single beam with an adjustable width to reach all neighbors that are not covered by transmissions of known forward nodes. Location information is used to calculate the angle and orientation of the transmission beam.

\section{Preliminaries}

We first give an antenna model that uses very few assumptions and accommodates a wide range of directional antenna systems. A simple scheme is then proposed for collecting 2-hop information, including relative directions of neighbors, without using location or AoA information. Then, we define efficient broadcasting using directional antennas as an optimization problem. The original selfpruning scheme [31] is reviewed as the first solution to this problem.

\subsection{Antenna Model}

Two techniques are used in smart antenna systems that form directional transmission/reception beams: switched beam and steerable beam [22]. Switched beam systems use fixed antenna patterns to transmit to or receive from specific directions. A simplified and yet popular antenna model for those systems is ideally sectorized [4], [9], [25], as shown in Fig. 1a. The effective transmission range of each node $v$ is equally divided into $K$ nonoverlapping sectors. Each node can switch on one or several sectors for transmission or reception. For example, to transmit to nodes $u$ and $w$, node $v$ can switch on both sectors 1 and 2. Aligned sectors are assumed in most existing protocols; that is, sector $i(i=1,2, \ldots, K)$ on all nodes point to the same direction. Steerable beam systems can adjust the bearing and width of a beam to transmit to or receive from certain neighbors. The corresponding antenna mode is an adjustable cone [10], [29], as shown in Fig. 1b. Most protocols also use omnidirectional transmission and reception modes. However, due to the different antenna gains in directional and omnidirectional modes, the effective transmission range in omnidirectional mode (represented by the dashed circle in Fig. 1a) is usually smaller than the area jointly covered by all directional transmission ranges. Both antenna models assume regular beam shapes for ease of computation, especially for estimating directions of neighbors based on location or AoA information. In practical systems, however, antenna beams have irregular shapes due to the existence of side lobes (as shown in Fig. 1c), which causes inaccurate estimations.

This paper uses an antenna model based on very few assumptions, such that the proposed directional broadcast protocol does not rely on a specific antenna type. Fig. 1d 


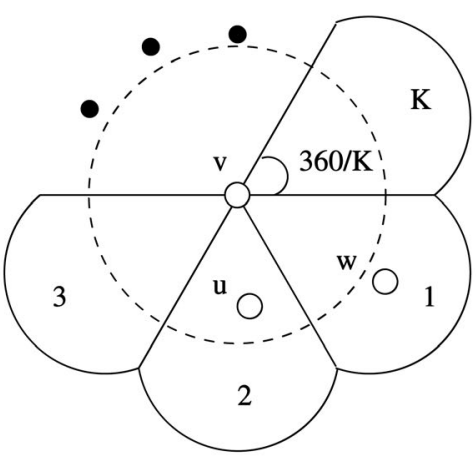

(a)

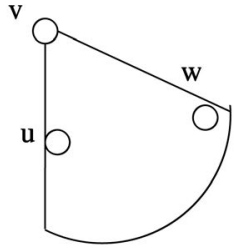

(b)

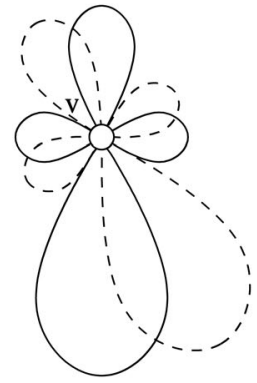

(c)

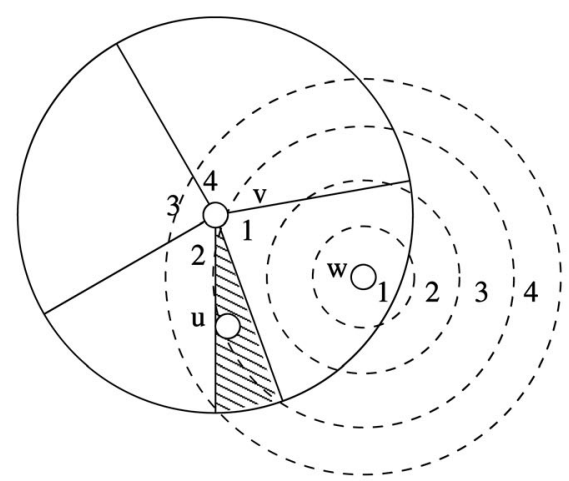

(d)

Fig. 1. Directional antenna models. (a) Ideally sectorized, (b) adjustable cone, (c) irregular beam pattern, and (d) a general model.

illustrates this model. We assume that each node can transmit and receive in $K$ directions with id's $1,2, \ldots, K$. In switched beam systems, each direction corresponds to a fixed antenna pattern. In steerable beam systems, a small set of beam settings, with different bearings and uniform or nonuniform widths, can be selected to cover a given neighborhood area. The shape of each direction does not have to be regular or aligned. For example, direction 1 of node $v$ is a cone, while direction 1 of node $w$ is a ring. The only constraint is that each direction must have a fixed size and shape. Directions can also be overlapping, as shown by the shadowed area between directions 1 and 2 of node $v$. Unlike in the ideally sectorized model, each node can only transmit in one direction at any moment. Multidirection transmission is emulated via sweeping [4], i.e., multiple directional transmissions in consequent time slots. Although this method incurs extra delay, it can be easily implemented on most directional antenna systems. In addition, single direction transmission has much longer transmission distance than omnidirectional or multidirection transmission. This enhanced per-hop transmission distance is essential for finding a shortest path with the minimum hop count in an on-demand route discovery process. There are two reception modes: the omnidirectional mode, where a node can receive from all neighbors, and the directional mode, where a node receives from neighbors in a single direction.

For each node $v, N_{i}(v)$ denotes the set of nodes within the transmission/reception range of $v^{\prime}$ s $i$ th direction, and $N(v)=N_{1}(v) \cup N_{2}(v) \cup \ldots \cup N_{K}(v)$ is $v^{\prime}$ s neighbor set. Note that a neighbor may appear in several directions when there is an overlapped area. For each neighbor $u$, its directions with respect to node $v$ is $D_{v \rightarrow u}=\left\{i \mid u \in N_{i}(v)\right\}$. For example, in Fig. 1d, $N(v)=\{u, w\}$, where $u, w \in N_{1}(v)$ and $u \in N_{2}(v)$. Therefore, $D_{v \rightarrow u}=\{1,2\}$ and $D_{v \rightarrow w}=\{1\}$. The network is viewed as a graph $G=(V, E)$, where $V$ is the set of nodes, and $E$ is the set of bidirectional links. A wireless link $(u, v) \in E$ if and only if $v \in N(u)$ and $u \in N(v)$. We assume the network is symmetric and connected via bidirectional links.

With the general antenna model, the application of the proposed protocol is not limited to directional antennas. In Fig. 1d, when node $w$ transmit with different power levels, the corresponding transmission range are rings with different radii. If we view those rings as directions, the corresponding protocol is an efficient broadcast scheme based on power control techniques.

\subsection{Directional Neighborhood Discovery}

Neighborhood information is collected via exchanging "Hello" messages among neighbors. A similar scheme was used in [22] to collect 1-hop information using AoA information. Here, we provide a simple scheme for collecting 2-hop information without using any location or AoA information. Olariu et al. [19] has recently proposed a similar scheme to create a coordinate system in a sensor network.

In directional neighborhood discovery, each node sends periodical "Hello" messages to its neighbors. Each "Hello" message is transmitted in all directions, with node id and direction id piggybacked in the message. By collecting "Hello" messages from its neighbors, each node $v$ can assemble its 1-hop information, including a list of its neighbors, and directions used by those neighbors to reach $v$. Note that the direction for $v$ to reach a neighbor $u$ is still unknown at that time. The 1-hop information, i.e., $N(v)$ and $D_{w \rightarrow v}: \forall w \in N(v)$ of each node $v$, is exchanged among neighbors in the next round of "Hello" messages. By assembling the 1-hop information of $v$ and its neighbors, node $v$ can construct its 2-hop information, which is a subgraph of $G$ derived from $v$ 's closed neighbor set $N[v]=N(v) \cup\{v\}$. Specifically, $v^{\prime}$ s 2-hop information provides the following: 1) for any two nodes $u$ and $w$ in $N[v]$, whether a link $(u, w)$ exists, and 2$)$ if such a link exists, the set of directions $D_{u \rightarrow w}\left(D_{w \rightarrow u}\right)$ that node $u(w)$ uses to reach node $w(u)$. Note that $v^{\prime}$ s 2-hop neighbors are excluded from the 2-hop information, because the direction from a 1-hop neighbor to any 2-hop neighbor is unknown.

In the above scheme, each "Hello" message is sent out $K$ times in $K$ directions at each node. In traditional neighbor discovery schemes using omnidirectional "Hello" messages, each message is sent only once. However, given the same neighborhood area, the bandwidth and energy consumption of each directional transmission is roughly $1 / K$ that of an omnidirectional transmission. The total cost of the directional neighborhood discovery is similar to that 
of the traditional scheme. This scheme also works when there are obstacles, as the neighbor and direction information is retrieved from real signal reception instead of being computed from an ideal antenna pattern. Collecting $k$-hop information with $k>2$ is possible, but will cause larger "Hello" messages and slower convergence. We assume that node movement, in terms of changing positions or turning on their axes, is relatively slow with respect to the "Hello" interval, so that 2-hop information collected at each node is up-to-date. We also assume that packet collision is avoided via an ideal MAC layer; otherwise, no broadcast protocol, including simple flooding, will guarantee full delivery. For clarity, we use ideally sectorized direction shapes in examples. Nevertheless, all results in this paper work for the general antenna model as shown in Fig. 1d.

\subsection{Efficient Broadcasting}

In ad hoc networks using omnidirectional antennas, for each broadcasting, some nodes (called forward nodes) are selected to forward the packet. In networks using directional antennas, each node selects some directions (called forward directions) to forward the packet. We define the forward scheme, $F$, as a function on $V$, where $F(v)$ is the set of $v^{\prime}$ s forward directions. Given $F$, we say a destination $d$ is reachable from a source $s$, if $s=d$ or there exists a forward path $P:\left(v_{1}=s, v_{2}, \ldots, v_{l}=d\right)$ satisfying that every node in $P$ forwards to the direction of its successor. That is, $D_{v_{j} \rightarrow v_{j+1}} \cap F\left(v_{j}\right) \neq \emptyset$ for $1 \leq j<l$. We say a forward scheme $F$ achieves full delivery if all nodes in the network are reachable from $s$.

Full delivery can be easily achieved via flooding, i.e., $F(v)=\{1,2, \ldots, K\}$ for all $v \in V$. For efficient broadcasting, the objective is to use a small number of forward directions to conserve bandwidth and energy consumption. For a given antenna model, we define the transmission cost of a forward scheme as $|F|=\sum_{v \in V}|F(v)|$, where $|F(v)|$ is the number of forward directions of node $v$, and the directional broadcasting problem as follows:

Efficient Broadcasting: Given a number of antenna directions $K$, network $G$, and source s, find the forward scheme $F$ that achieves full delivery with minimum transmission cost $|F|$.

Efficient broadcasting using omnidirectional antennas is a special case of the above problem with $K=1$. Broadcasting in ad hoc networks with a minimal number of forward nodes is equivalent to finding a minimum connected dominating set (CDS), which is known to be NP-complete. The efficient broadcasting problem with a particular $K \geq 2$ in a geometric graph is conjectured to be NP-complete. A distributed broadcast algorithm is localized, if each node selects its forward direction based on local neighborhood information (e.g., 2-hop information). Our objective is to find a localized solution with a low average transmission cost.

We first review the omnidirectional self-pruning (OSP) as a trivial solution to the above problem. In OSP, each node computes the coverage of its neighborhood after receiving the packet from one or several known forward nodes. In node $v$ 's local view, a node $w$ is covered if: 1) $w$ is a known forward node, 2) $w$ is a neighbor of a known forward node, or 3) $w$ is a neighbor of a covered node with a higher id than $v$. If $v$ has uncovered neighbors, it becomes a forward node and transmits in all directions; otherwise, it does nothing. It was proved in [31] that OSP guarantees full delivery.

\section{Proposed Scheme}

For efficient broadcasting using directional antennas, we propose directional self-pruning (DSP) to replace the omnidirectional self-pruning (OSP) scheme. In DSP, forward nodes can switch off transmissions in directions of covered neighbors to conserve broadcast cost. However, the definition of covered node must be enhanced to maintain full delivery without being overly conservative. OSP can be viewed as a special case of DSP with $K=1$. Compared with OSP, DSP uses slightly more forward nodes, and far fewer forward directions. We prove that DSP ensures full delivery, and the average number of forward nodes in DSP is within a constant factor of the minimal value in an optimal solution.

\subsection{Why OSP to DSP Is Nontrivial}

Intuitively, OSP can be improved using directional antennas. It seems that each forward node only needs to transmit in directions of uncovered neighbors; other directions can be switched off to conserve transmission cost. However, the task of fitting the original self-pruning scheme in a directional transmission model is not straightforward. Being too aggressive and switching off too many directions cause broadcast failures. On the other hand, being overly conservative brings about unnecessarily low pruning efficiency.

Fig. 2a shows a failed broadcast process in an "optimized" version of OSP which transmits only in directions of uncovered neighbors. First, source node 1 transmits the broadcast packet to its neighbors 4 and 5 . In node 4's local view, all nodes, except node 2 in direction 1, are covered. Therefore, node 4 forwards in direction 1 only. This packet is received by neighbors 2 and 5 , but not by nodes 3,6 , and 7. If node 5 overhears both transmissions of nodes 1 and 4 , it has two known forward nodes in its local view, and the third neighbor, node 6 , is covered by node 4 . It becomes a nonforward node because all neighbors are covered. Similarly, node 2 also becomes a nonforward node. As a result, the broadcasting stops after two transmissions, and nodes $3,6,7$, and 8 have never received the broadcast packet.

One may argue that the above problem can be solved by using a more cautious rule to identify covered nodes. In the above example, even if node 6 is a neighbor of a forward node, it should not be considered as covered because it has not received the packet from node 4 . However, using such a definition of coverage is overly conservative which causes unnecessary transmissions. As shown in Fig. 2b, when neighbors of a forward node in its nonforward directions are not considered covered, nodes 2, 3, 5, 6, and 7 become forward nodes and achieve full delivery. However, the transmission of node 2 is unnecessary; all of its neighbors have been covered by node 3 . In the following section, we define a new coverage rule to avoid those problems. 


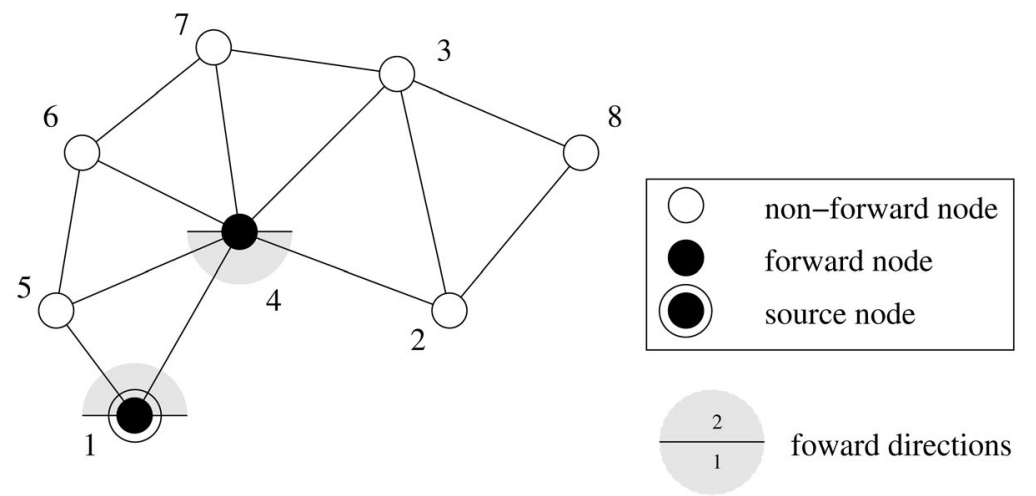

(a)

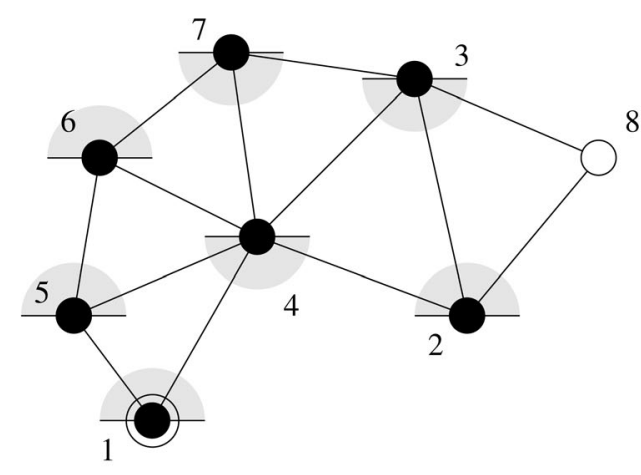

(b)

Fig. 2. Problems in converting OSP to DSP. (a) A failed broadcast process and (b) an overly conservative broadcast process.

\subsection{Directional Self-Pruning}

In DSP, each forward node $u$ includes its forward directions, $F(u)$, in the broadcast packet. Based on the refined definition (called the directional coverage rule), a node $w$ in node $v$ 's local view is covered if and only if:

1. $w$ is a known forward node,

2. $\quad w$ is a neighbor of a known forward node $u$ and $w$ is within one of $u^{\prime}$ s forward directions (i.e., $D_{u \rightarrow w} \cap F(u) \neq \emptyset$ ), or

3. $w$ is a neighbor of a covered node with a higher id than $v$.

As shown in Fig. 3, a covered neighbor is either a forward node or connected to a forward node via a replacement path $\left(u, w_{1}, w_{2}, \ldots, w_{m}, w\right)$, where $i d\left(w_{i}\right)>i d(v)$. Two scenarios exist: 1$) i d(u)<i d(v)$, then $w_{1}$ must be within a forward direction of $u$ in order to apply term 2; 2) $i d(u)>i d(v)$, then $w_{1}$ can be out of $u$ 's forward directions by applying term 3 .

Based on the new rule, node 5 in Fig. 2 can no longer view node 6 as covered, because node 4 has not transmitted in direction 2 and, in addition, node 4 has a lower id than node 5 . Therefore, node 5 becomes a forward node. Similarly, nodes 6,7 , and 3 become forward nodes and ensure full delivery. On the other hand, node 2 can still view nodes 3 and 8 as covered, because both nodes are connected via a replacement path to node 4 , which has a higher id than node 2 . Therefore, node 2 becomes a nonforward node.

\section{Algorithm 1 Directional Self Pruning}

(DSP, at each node $v$ )

1: Compute the set $C$ of covered nodes based on directional coverage rules.

2: If $N(v) \subseteq C$, then $v$ becomes a nonforward node (i.e., $F(v)=\emptyset)$.

3: Otherwise, $v$ becomes a forward node, and

$$
F(v)=\left\{d_{v \rightarrow w} \mid w \in(N(v)-C)\right\} .
$$

Algorithm 1 gives the DSP algorithm. In line 1, it uses the new coverage rule to compute the set of covered nodes. In line 3 , the data packet is transmitted only in directions with uncovered nodes, instead of all directions. For each uncovered neighbor $w$, at least one direction $d_{v \rightarrow w} \in D_{v \rightarrow w}$ becomes a forward direction in $F(v)$. If there are overlapping directions, an uncovered node may appear in different directions (i.e., $\left|D_{v \rightarrow w}\right|>1$ ). In this case, a greedy heuristic algorithm for the set coverage problem [7] can be used to select a minimum $F(v)$ that covers all nodes in $N(v)-C$. The source node is always a forward node, but it can switch off transmissions in those "empty" directions without neighbors. Note that OSP can be viewed as a special case of DSP with $K=1$.

Fig. 4 illustrates DSP, which uses four forward nodes and eight forward directions. The source node 1 transmits in all four directions. Node 2 transmits in only one direction, because in its local view (as shown in Fig. 4b), all neighbors except node 7 are covered. There is an uncovered node 8 in node 9's local view (as shown in Fig. 4c). Therefore, node 9 becomes a forward node and transmits in direction 1 .

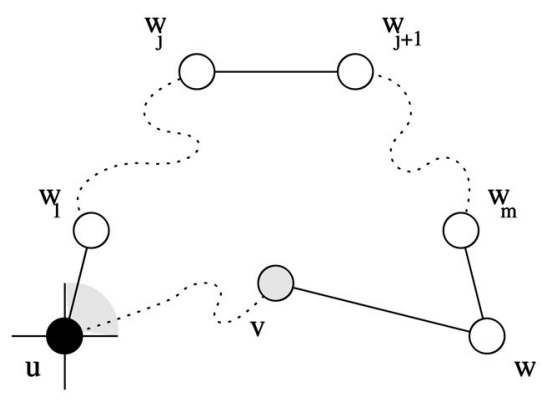

(a)

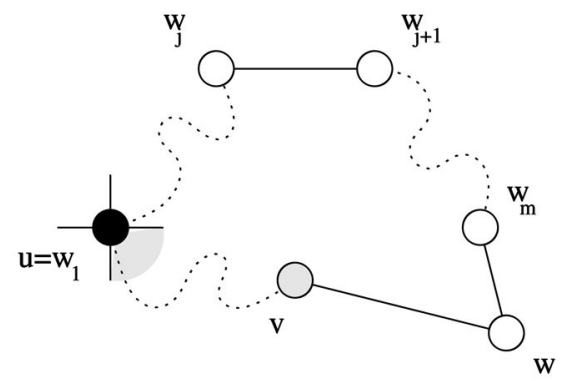

(b)

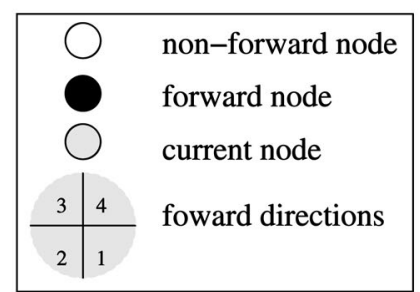

non-forward node

forward node

foward directions

Fig. 3. Two scenarios of applying term 3 of the directional coverage rule. 


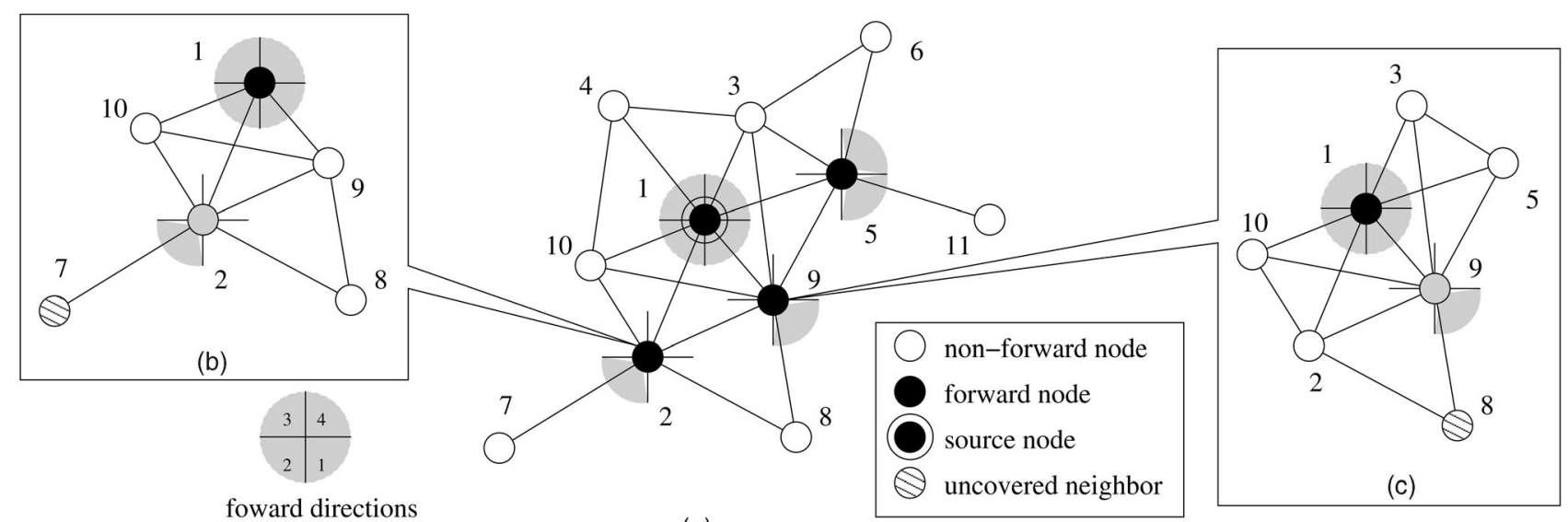

foward directions

(a)

Fig. 4. Directional self-pruning (DSP) in a network with 10 nodes and four directions. (a) An efficient broadcasting, (b) node 2's local view, and (c) node 9's local view.

Similarly, node 5 has two uncovered nodes 6 and 10, and transmits in directions 3 and 4 .

\subsection{Properties of DSP}

In this section, we prove the correctness of DSP and provide a constant probabilistic bound on the number of forward nodes used by DSP. The following theorem guarantees that every node eventually receives the broadcast packet.

Theorem 1. The forward scheme determined by DSP achieves full delivery.

Proof. By contradiction, suppose there is at least one node that is unreachable from the source $s$. Let $U$ be the set of "border" nodes that 1 ) is reachable from the source node and 2) has an unreachable neighbor. $U$ is not empty in a connected network. Let $v$ be the node with the highest id in $U$, and $w$ an unreachable neighbor of $v$. Since $v$ has not transmitted in $w^{\prime}$ s direction, node $w$ must be covered in $v$ 's local view. However, we show that $w$ cannot be covered, as none of the three terms in the directional coverage rule applies:

1. $w$ is a known forward node, which implies that $w$ is reachable from $s$.

2. $w$ is a neighbor of a known forward node $u$ that has transmitted in $w^{\prime} s$ direction. In this case, $w$ is reachable from $s$ via a forward path through $u$.

3. $w$ is a neighbor of a covered node with a higher id than $v$. There are only two possible scenarios, as shown in Fig. 3. In both cases, the unreachable node $w$ is connected to a reachable node $w_{1}$ via a path $P:\left(w_{1}, w_{2}, \ldots, w_{m}, w\right)$, where each $w_{i}(1 \leq i \leq m)$ has a higher id than $v$. There is at least one node $w_{j}$ in $P$ that has an unreachable neighbor $w_{j+1}$ (here, we view $w$ as $w_{m+1}$ ). That is, $w_{j} \in U$, which contradicts the assumption that $v$ has the highest id in $U$.

Self-pruning protocols, including both OSP and DSP, are dynamic algorithms, where the forward status is determined during the broadcast process, and forward nodes form a source-dependent CDS. In certain scenarios, a static algorithm that maintains a source-independent CDS are preferable. Dynamic algorithms produce a smaller CDS than static algorithms, as they use dynamic (forward node) information to improve the pruning efficiency. Static algorithms are usually used to form a virtual backbone of the network. A typical static algorithm is Rule $k$ [6], where a node is a nonforward node if all its neighbors can be connected via several nodes with higher id's, and a forward node otherwise. Rule $k$ has been shown as a special case of OSP [31]; if a node can be pruned by Rule $k$, it can also be pruned by OSP.

It was proven in [6] that Rule $k$ has a constant probabilistic bound. That is, the expected number of forward nodes selected by Rule $k$ is $O(1)$ times that in an optimal solution. As OSP has fewer forward nodes than Rule $k$, the above probabilistic bound also holds for OSP. We cannot, however, apply the same bound directly to DSP, because DSP may use more forward nodes than OSP. The following theorem shows that, in a random ad hoc network, the expected ratio of the forward node number in DSP to the minimal value in an optimal algorithm is still a constant. For simplicity, we assume all nodes are uniformly distributed in a boundless area. The case in a bounded area can be done in a similar manner to the proof in [6].

Theorem 2. Given an ideally sectorized antenna model with $K$ sectors, the expected number of forward nodes determined by $D S P$ is $O(1)$ times that in an optimal solution in random ad hoc networks.

Proof. We first prove that the average number of forward nodes in each unit area is a constant. The basic idea is that, if a few nodes with the highest id's occupy certain "good" positions, other nodes with lower id's will become nonforward nodes, no matter how many nodes are in this area.

Consider small squares with area size $A$. By putting $m$ squares together in a pattern illustrated in Fig. 5, if a directional beam (represented by a cone) of a known forward node reaches any node in the central (black) square, this beam will also contain some peripheral squares. Therefore, all nodes in those "covered" (gray) squares are within a forward direction of the known forward node. Such a layout is always possible for a given $K$, if we make $A$ sufficiently small and $m$ sufficiently large. Let $v$ be a node in the central square; $v$ will become a nonforward node if each of the $m$ squares contains a node with a higher id than $v$ : No 


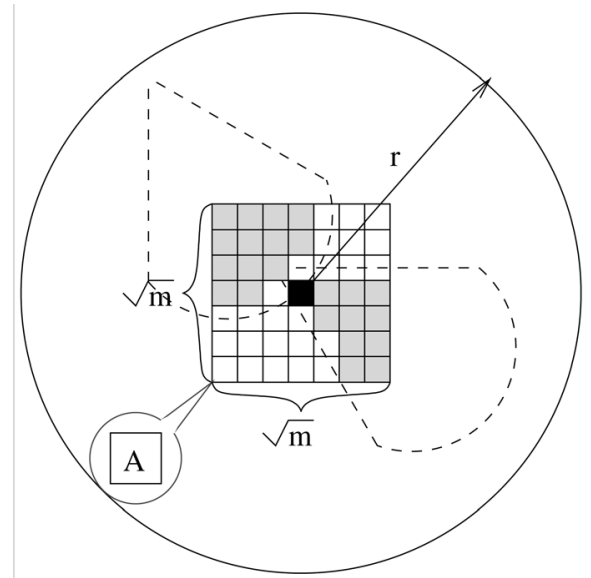

Fig. 5. Proof of Theorem 2.

matter where the known forward node is in $v^{\prime}$ s local view, at least one of those $m$ nodes are within the forward direction of the known forward node and considered covered. Consequently, all those $m$ nodes with higher id's than $v$ are also covered, because they are connected to that node. Also, all neighbors of $v$ (within the large circle representing the combined transmission range of nodes in the central square) are considered covered because they are neighbors of those $m$ nodes.

Let $n$ be the number of forward nodes in the central square, and $n^{\prime}$ be the number of nodes in all $m$ squares with id's larger than or equal to these forward nodes. Based on the above discussion, some of the $m$ squares must be "empty," i.e., do not contain any of the $n^{\prime}$ nodes; otherwise, the forward node with the minimal id will be a nonforward node, which is a contradiction. As the $n^{\prime}$ nodes are uniformly distributed among $m$ squares with independent distribution, the probability

$$
\operatorname{Pr}\{n \geq x\} \leq \operatorname{Pr}\left\{X \mid n^{\prime} \geq x\right\} \leq m\left(1-\frac{1}{m}\right)^{x},
$$

where $X$ represents event "at least one of the $m$ squares is empty" and the expected number of forward nodes within area $A$ is

$$
E[n]=\sum_{x=1}^{\infty} \operatorname{Pr}\{n \geq x\} \leq m \sum_{x=1}^{\infty}\left(1-\frac{1}{m}\right)^{x}=m^{2} .
$$

In an optimal solution, a forward node can cover at most $\pi r^{2} / A$ squares, where $r$ is the maximal transmission range of each node. Therefore, the expected ratio of the number of forward nodes in DSP to that in an optimal solution is at most $\pi r^{2} m^{2} / A=O(1)$, as $r, m$ and $A$ are constants for a given set of fixed beam patterns.

Both $m$ and $A$ depend on $K$. The specific value of probabilistic bound increases as the number of directions increases. Whether a bound exists on the number of forward directions remains an open problem.

\section{EXtensions}

We consider two special cases of DSP: directional self-pruning with shortest paths (DSP-SP) that supports shortest path routing in reactive routing protocols, and directional selfpruning with directional reception (DSP-DR) that uses the directional reception mode to further reduce the broadcast redundancy.

\subsection{DSP with Shortest Paths}

In order to support shortest paths routing in reactive routing protocols, it is desirable for the RREQ packet to arrive at the destination along a shortest path with minimal hop count. By using directional transmissions in broadcasting, DSP has already achieved maximal per-hop transmission distance. In order to minimize the number of hops, we propose the shortest path extension of DSP. In this extension (called DSP-SP), the scope of "covered nodes" is restricted by applying a distance-related constraint in term 3 of the directional coverage rule. In the original term 3 , a neighbor $w$ is viewed as covered if it connected to a known forward node $u$ via a replacement path. In DSP-SP, the length of the replacement path is restricted within two hops. That is, $w$ is considered as covered only when it is a neighbor of $u$, or a neighbor's neighbor of $u$. Here, we assume the propagation delay of a broadcast packet is proportional to the number of hops it travels. To maintain this assumption, each node determines its forward directions immediately after receiving the first copy of a broadcast packet. No backoff delay is used to find more known forward nodes. In the worst case, when the propagation delay is nonuniform at each hop, DSP-SP is as good as blind flooding in terms of finding shorter paths.

Using DSP-SP, the broadcast process in Fig. 4 will be the same. It is because in each local view of this sample network, there is only one known forward node, and the length of each replacement path that connects a covered node to the known forward node is at most 2. For example, in node 2's local view, both covered nodes 9 and 10 are within 1 hop of the known forward node 1, and covered node 8 is within 2 hops of node 1 . In most cases, however, fewer nodes are viewed as covered in DSP-SP than in DSP, which yields more forward nodes and forward directions. The probabilistic bound on the number of forward nodes in DSP does not apply to DSP-SP. On the other hand, the transmission cost of DSP-SP is comparable to the omnidirectional self-pruning [31], which is significantly lower than similar schemes using omnidirectional antennas [23], [32].

Theorem 3. The forward scheme determined by DSP-SP achieves full delivery, and, in addition, the broadcast packet is transmitted to each node via a shortest path, if each hop of transmission takes the same amount of time.

Proof. By induction on source node $s$ 's $i$-hop neighbors. The theorem is true for $i=1$, because the source transmits in all directions, and all 1-hop neighbors of $s$ receive the broadcast packet in the first round. Suppose all $i$-hop neighbors of $s$ receive the broadcast packet in the $i$ th round $(i \geq 1)$. Let $d$ be any $(i+1)$-hop neighbor of $s, C$ be the set of $d$ 's neighbors that are also $s$ 's $i$-hop neighbors, and $v$ be the node with the highest id in $C$. When $v$ receives the broadcast packet from a $(i-1)$-hop neighbor $u$ of $s$, it views $d$ as uncovered in its local view, because any replacement path connecting $u$ and $d$ with length 2 must use an intermediate node from $C$, but no node in $C$ has a 
higher id than $v$. Therefore, $v$ must forward in the direction of $d$, and $d$ will receive the packet in the $(i+1)$ th round.

\subsection{DSP with Directional Reception}

The directional reception mode is very effective in reducing interference and improving the quality of the incoming signal. In addition, if some nodes have decided to receive from only a subset of its neighbors, this information can be utilized in a schedule-based MAC protocol [2] to improve channel utilization. Omnidirectional reception has been assumed in existing broadcast protocols. In the second special case of DSP, we show that a majority of nodes in the network can direct its reception beam to a single neighbor for better SNR and spatial reuse ratio.

In DSP-DR, a time division scheme similar to that in [2] is used. Each "Hello" interval is divided into a small time portion for "Hello" message exchanges, and a large time portion for data transmission. In the first time portion, all nodes collect 2-hop information and run a localized CDS formation algorithm, such as Rule $k$ [6]. Nodes in the CDS form a virtual backbone that connects all nodes in the network. After the virtual backbone formation, every nonvirtual backbone node selects one virtual backbone neighbor as its unique dominator. When there are multiple neighboring backbone nodes, the one with the highest id is selected. At the beginning of the second time portion, all virtual backbone nodes use the omnidirectional reception mode, while each nonvirtual backbone node uses the directional reception mode and points its transmission/reception beam toward its dominator. In a broadcasting, each virtual backbone node applies the DSP algorithm to determine its forward status and forward directions, while all nonvirtual backbone nodes, except the source, are nonforward nodes. Compared with the original DSP, local views of virtual backbone nodes are sparsified. Only one link is preserved for each nonvirtual backbone node $v$ : the one connecting the $v$ and its dominator. All other adjacent links of $v$ are removed from local views of neighboring nodes.

Theorem 4. The forward scheme determined by DSP-DR achieves full delivery and the expected number of forward nodes determined by DSP-DR is $O(1)$ times that in an optimal solution.

Proof. DSP-DR is equivalent to broadcasting with DSP in a sparsified topology after removing links between nonvirtual backbone nodes and their nondominator neighbors. When the virtual backbone nodes form a CDS, and all nonvirtual backbone nodes are connected to the virtual backbone, the sparsified topology is still connected, and the resultant forward scheme guarantees full delivery.

The number of forward nodes in DSP-DR is at most the number of virtual backbone nodes plus 1 . Because the expected number of virtual backbone nodes determined by Rule $k$ is $O(1)$ times that in an optimal solution [6], this probabilistic bound also applies to DSP-DR.

\section{Simulation}

We evaluate DSP via two groups of simulations. The first group focuses on the pruning efficiency of the proposed algorithms and is conducted using a custom simulator in ideal networks without packet losses. In the second group, DSP is compared with several existing directional broadcast algorithms, in terms of both efficiency and reliability, in more realistic networks using the network simulator $n s 2$ [8].

\subsection{Simulation in Ideal Networks}

We first simulated DSP, OSP, and blind flooding in ideal networks without packet collision, channel contention, or node mobility. Simulations are conducted in random networks with 30-160 nodes deployed in a $1,000 \mathrm{~m} \times$ $1,000 \mathrm{~m}$ area. All nodes have a transmission range of $250 \mathrm{~m}$ and an ideally sectorized antenna pattern with $K$ sectors $(2 \leq K \leq 16)$. The following metrics are compared:

1. number of forward nodes,

2. normalized transmission cost $|F| / K$,

3. redundancy ratio (i.e., average number of redundant receptions per node), and

4. average routing distance in hops.

The first two metrics measure the efficiency of a broadcast algorithm. Metric 3 is an indicator of robustness and the level of interference. Metric 4 corresponds to the average end-to-end delay and the expected length of routes discovered in a reactive routing protocol. Together, they measure the broadcast quality. The 90 percent confidence intervals of these metrics are within \pm 1 percent.

Fig. 6 illustrates omnidirectional and directional selfpruning in a random network with 50 nodes. OSP (shown in Fig. 6a) uses 21 forward nodes. Its normalized transmission cost is also 21. Its redundant ratio is 2.74 . The average routing distances is 3.70. DSP (shown in Fig. 6b) uses 22 forward nodes, 34 forward directions, and a normalized transmission cost of 8.5 . Its redundant ratio is 1.56 . The average routing distances is 3.72 .

Efficiency. Fig. 7a and Fig. $7 \mathrm{~b}$ compare the broadcast cost of DSP and OSP. Previous simulation results shown that OSP is more efficient than most existing deterministic broadcast protocols [31], which in turn are more efficient than probabilistic protocols [30]. The normalized transmission cost of DSP with $K=2,4,8$, and 16 is about 70 percent, 55 percent, 45 percent, and 35 percent that of OSP. Although DSP has slightly (5-10 percent) more forward nodes than OSP, each forward node uses only a few forward directions. The portion of nonforward directions increases as more directions are used to create finer divisions. On the other hand, the gain in broadcast efficiency is not a linear function of $K$. Considering the complexity of forming many beam patterns, using $K=4$ or $K=8$ is good enough to conserve bandwidth and energy consumption.

Quality. Fig. 7c shows the redundancy of blind flooding, OSP, and DSP. The redundancy ratio of blind flooding increases as the number of nodes increases, while redundant ratios of the self-pruning schemes remain low. Specifically, the redundant ratio of OSP is about 4 , and that of DSP with $2 \leq K \leq 16$ is between 1.8 to 3.5. As DSP has very low redundancy with a larger $K$, it is very efficient at conserving bandwidth. On the other hand, it may suffer a reliability problem in situations with heavy packet losses. In such a case, either a small $K$ or some reliability mechanisms should be used. 


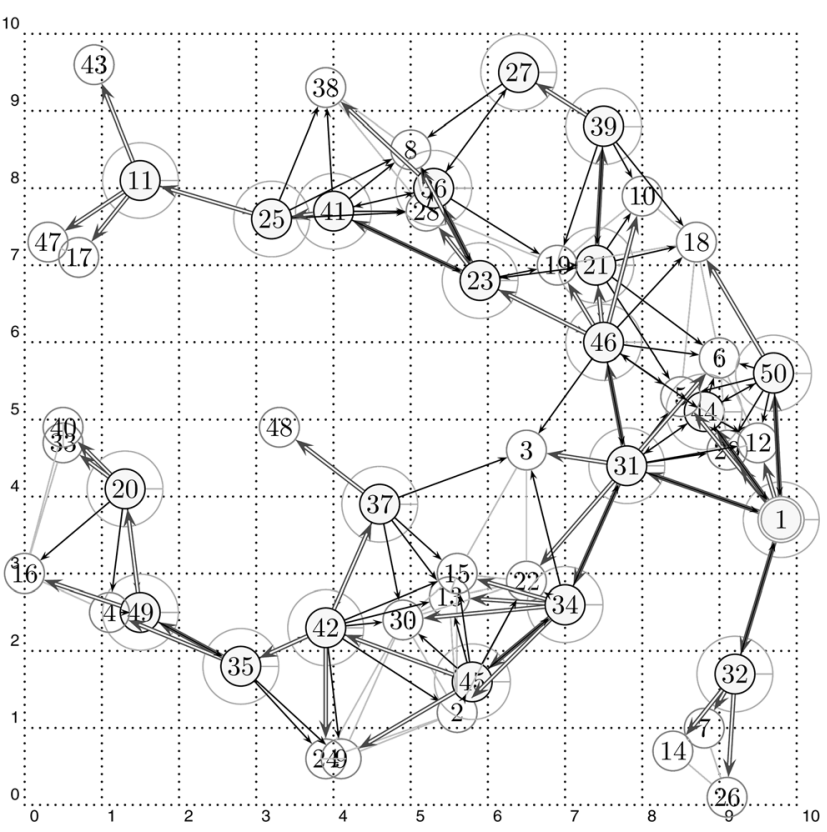

(a)

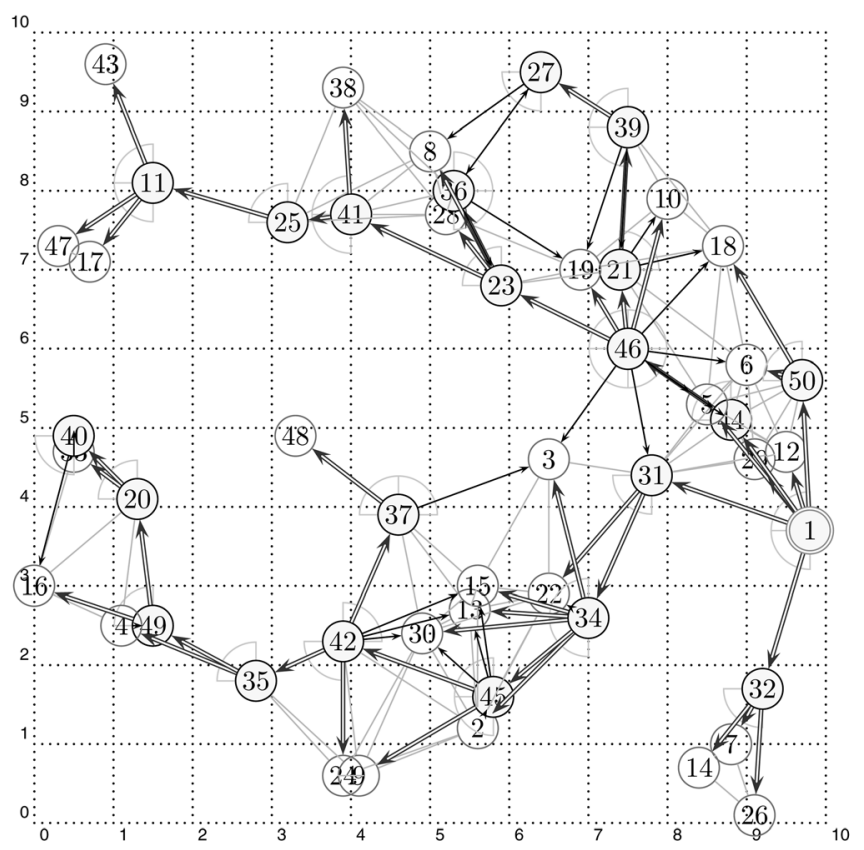

(b)

Fig. 6. Sample broadcast processes from source node 1. (a) OSP and (b) DSP $(K=4)$.

Fig. 7d compares average routing distances. The average routing distance of blind flooding is about 20 percent shorter than those of self-pruning algorithms. If either OSP or DSP is used to disseminate RREQ in route discovery, the resultant route is expected to be 20 percent longer than the one discovered via blind flooding. The difference between different self-pruning algorithms is very small.

Extensions. Fig. 7e and Fig. 7f compare DSP-SP and DSPDR with DSP, OSP, and a variation of OSP that preserves shortest paths (OSP-SP). The number of sectors is $K=8$.
Both DSP-SP and OSP-SP have the shortest average routing distance (the same as that of blind flooding). The penalty is that their transmission cost and broadcast redundancy are doubled, compared with the general DSP and OSP protocols that have a longer routing distance. On the other hand, the transmission cost of DSP-SP is smaller than OSP, and its broadcast redundancy is similar to that of OSP. Replacing OSP with DSP-SP for a route discovery can reduce routing distance without increasing overhead.

The normalized transmission cost of DSP-DR is similar to that of the general DSP. However, the redundancy ratio of DSP-DR is very close to 1 (i.e., no redundancy), which is significantly smaller than the general DSP. This is because most nodes in DSP-DR are nonvirtual backbone nodes. In most cases, a nonvirtual backbone node receives the broadcast packet only once from its dominator. Since each node can only receive from one sender at a time, fewer redundant receptions in DSP-DR implies less interference and higher spatial channel reuse ratio.

The above simulation results can be summarized as follows:

1. DSP uses slightly more forward nodes than OSP, but has a much lower bandwidth and energy consumption.

2. The redundant ratio of DSP is 50 percent to 89 percent that of OSP.

3. The average routing distance of DSP is very close to that of OSP, and is about 20 percent longer than the optimal distance.

4. DSP-SP has the same routing distance as blind flooding, while its overhead is similar to that of OSP.

5. DSP-DR achieves a very low (near 1) redundancy ratio using directional reception mode.

\subsection{Simulation in Realistic Networks}

We also simulated DSP on $n s 2.1 b 9$ to study its efficiency, overhead, delivery ratio, and delay as functions of network density, collision, and mobility. Our simulations use the directional antenna model and an enhanced IEEE 802.11 MAC layer provided by the enhanced network simulator (TeNs) [24], and the steady state, random waypoint mobility model [18]. The simulated ad hoc network is also deployed in a $1,000 m \times 1,000 m$ area with $30-160$ nodes. All nodes have a transmission range of $250 \mathrm{~m}$, an omnidirectional antenna for reception, and four directional antennas with an ideally sectorized radiation pattern for transmission (i.e., $K=4$ ). All nodes share a single $2 M b$ channel. The traffic load is 1-10 packets per second ( $p p s$ ), with a packet size of 64 bytes. A higher traffic load has not been used because of a defect in the current MAC layer, as will be discussed later. The "Hello" message interval is $1 s$. The average node moving speed varies from 1 to $25 \mathrm{~m} / \mathrm{s}$.

Two existing schemes are simulated for comparison: 1) The probabilistic approach proposed by $\mathrm{Hu}$ et al. ( $\mathrm{HHH})$ [9]. In $\mathrm{HHH}$, the source node designates one neighbor in each direction as a forward node. Upon reception, a forward node forwards in all directions, except the direction of the source, and designates one neighbor in each of these directions as a forward node. 2) The directional neighbor elimination approach proposed by Shen et al. (SHJ) [25]. In SHJ, each sender $u$ piggybacks its 


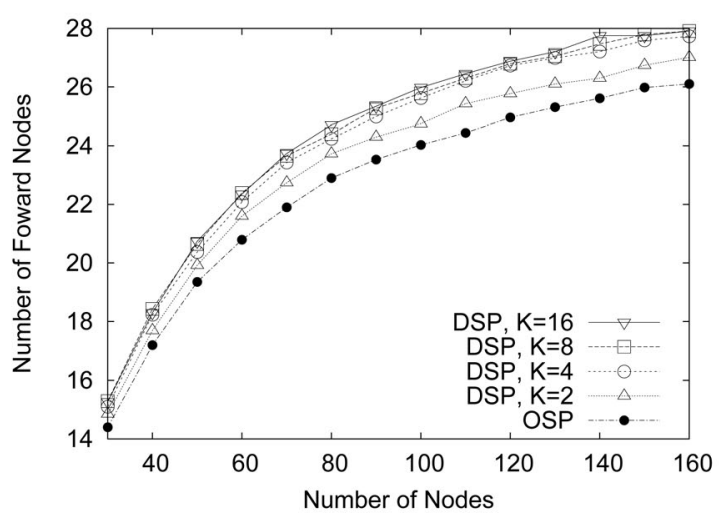

(a)

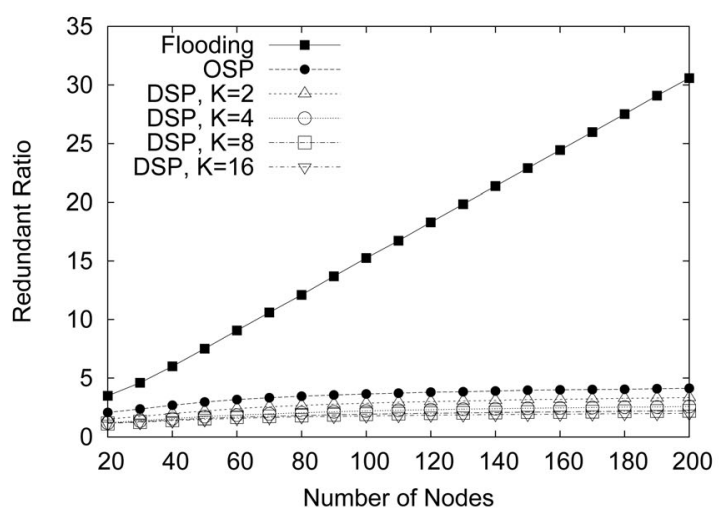

(c)

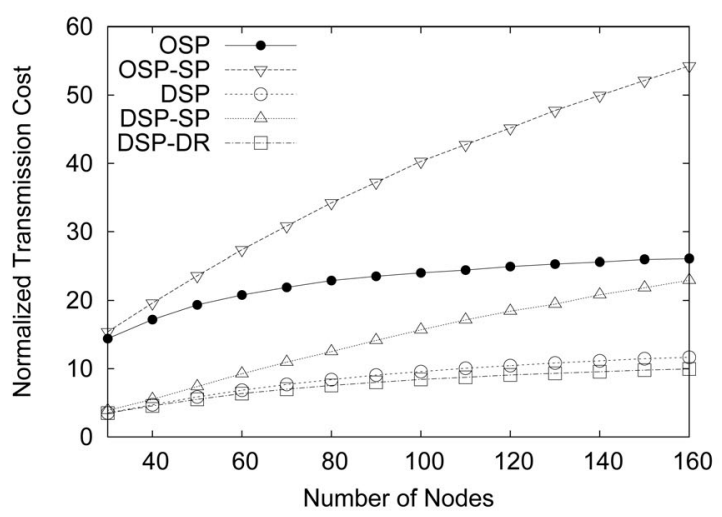

(e)

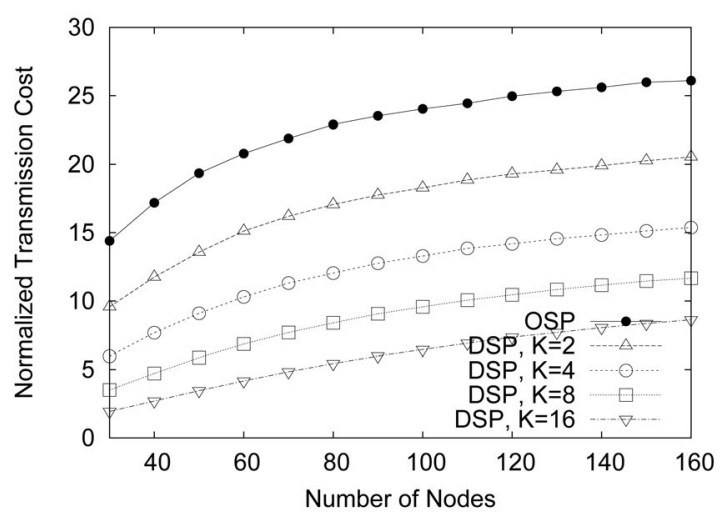

(b)

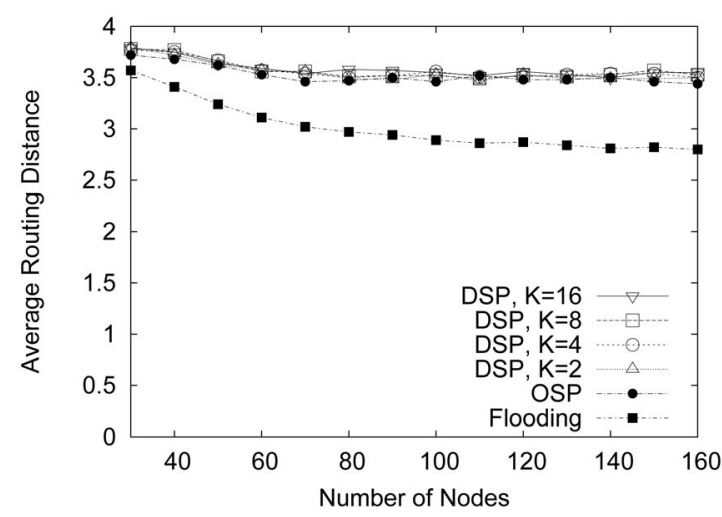

(d)

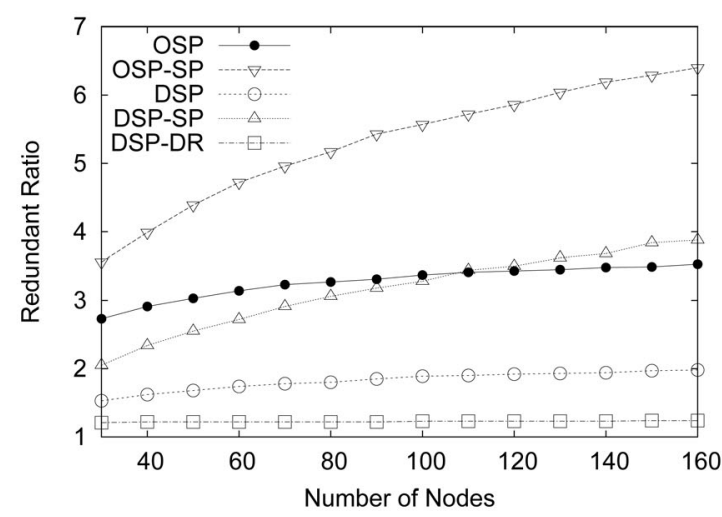

(f)

Fig. 7. Simulation results in ideal networks. (a) Forward node number. (b) Normalized broadcast cost. (c) Redundancy ratio. (d) Average routing distance. (e) Normalized cost of DSP extensions. (f) Redundancy ratio of DSP extensions.

neighbor set $N(u)$ into the broadcast packet. When a node $v$ receives this packet, it forwards the packet to only those "uncovered" directions $i$ satisfying $N_{i}(v)-N(u)-\{u\} \neq \emptyset$. This scheme was shown to be more efficient than other schemes in [25]. Both $\mathrm{HHH}$ and SHJ require 1-hop AoA information. SHJ guarantees full delivery in ideal networks, while $\mathrm{HHH}$ does not. Two omnidirectional broadcast schemes, OSP and Flooding, are also simulated. In these schemes, each forward node transmits the broadcast packet in all four directions.
Density. Figs. $8 \mathrm{a}, 8 \mathrm{~b}$, and $8 \mathrm{c}$ show performance of simulated protocols when the network population varies from 30 to 160 . The average node speed of a moving node is $1 \mathrm{~m} / \mathrm{s}$ and the traffic load is 10pps. Fig. 8a gives the efficiency in terms of average percentage of forward directions per broadcasting. Flooding has near 100 percent forward directions (some nodes may not receive the packet due to collision and do not forward). SHJ is better than Flooding, but less efficient than other schemes. The underlying neighbor elimination scheme [14] requires direct 


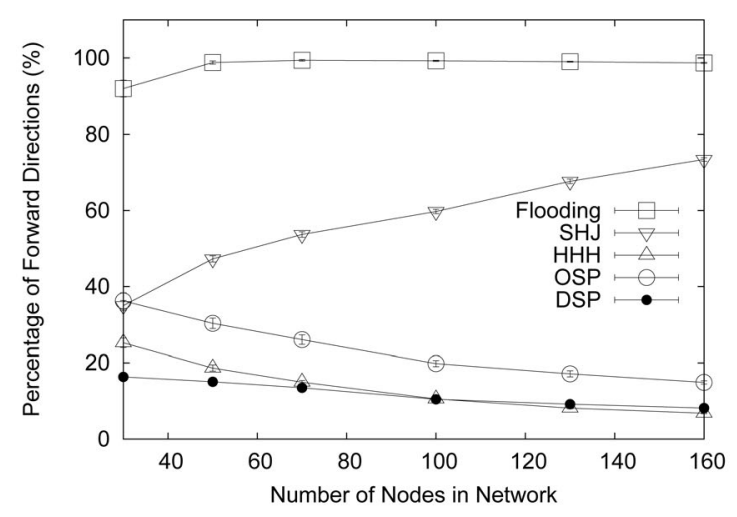

(a)

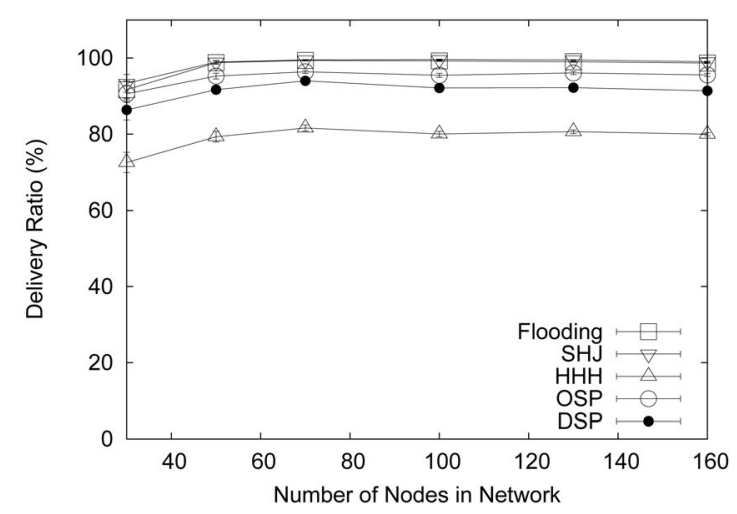

(c)

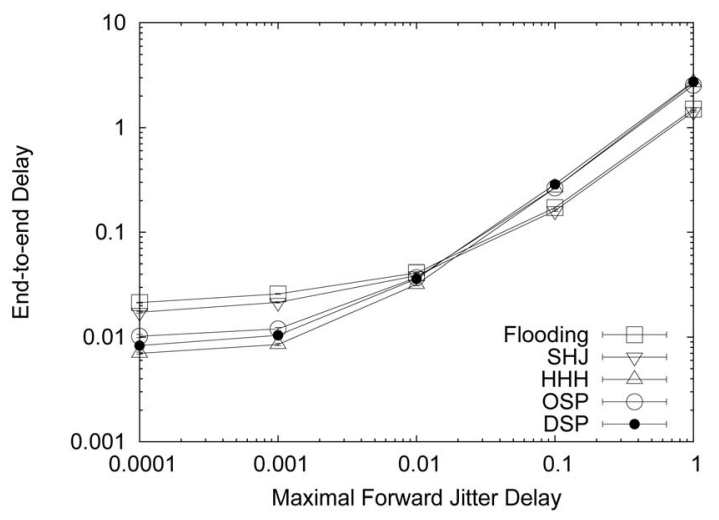

(e)

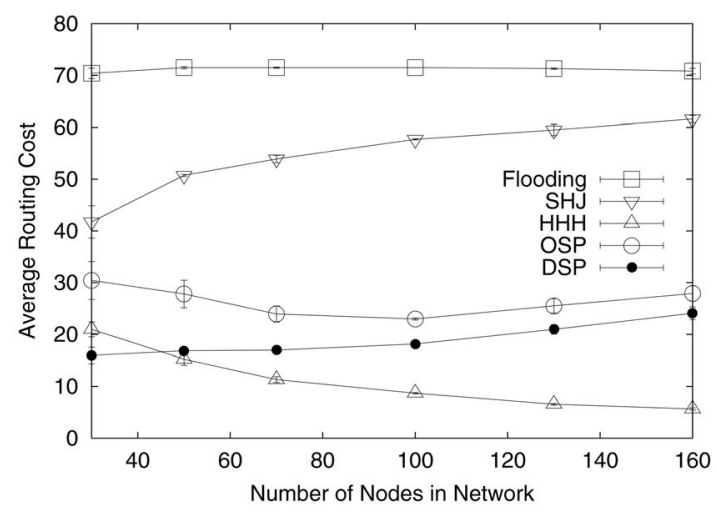

(b)

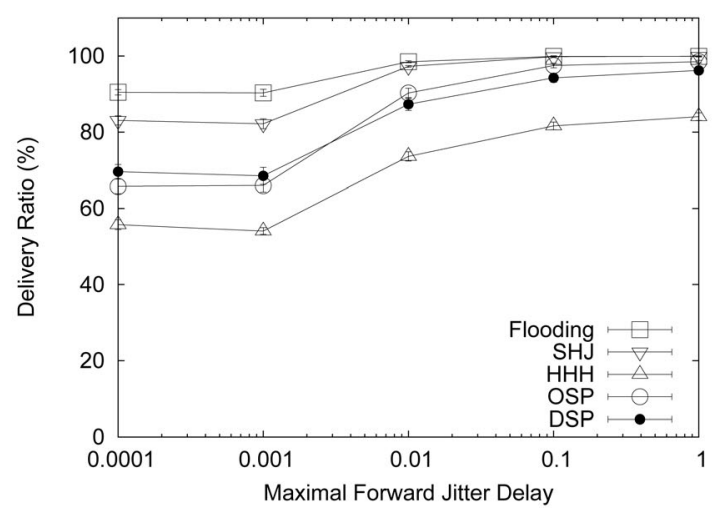

(d)

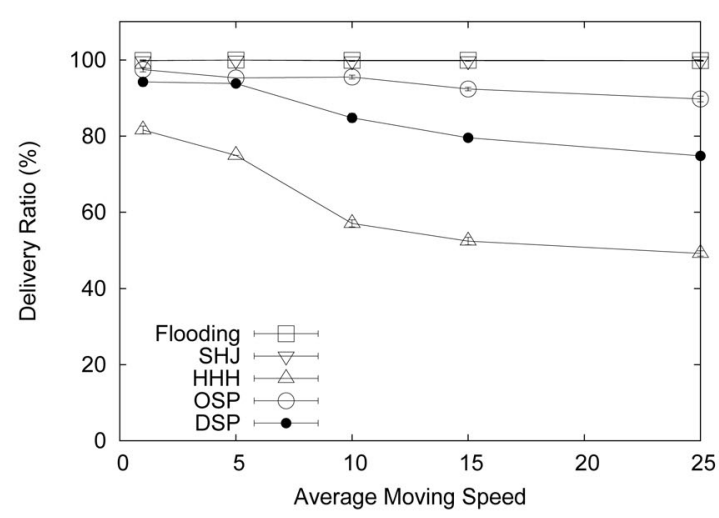

(f)

Fig. 8. Simulation results in realistic networks. (a) Fraction of forward directions. (b) Amortized broadcast overhead. (c) Delivery versus network size. (d) Delivery versus forward jitter (\$D_\{max $\}$ ). (e) End-to-end delay. (f) Delivery versus mobility.

coverage, which is less effective in dense networks. Both DSP and HHH are more efficient than OSP. DSP is better than $\mathrm{HHH}$ under most scenarios, and $\mathrm{HHH}$ is slightly better in very dense networks.

Fig. 8b compares amortized broadcast overhead in terms of average bytes sent to the MAC layer per direction. The result is similar to that in Fig. 8a with one exception: $\mathrm{HHH}$ has lower overhead than DSP in networks with more than 50 nodes. The reason is that HHH has a fixed "Hello" message size, while "Hello" messages in DSP contain a list of neighbors, and have a variable size increasing with network density. Nevertheless, both DSP and HHH have similar number of packet transmissions. If position information is available, DSP can also use a fixed "Hello" size and a lower overhead than HHH.

Fig. 8c compares reliability in terms of delivery ratio. Flooding and SHJ achieve almost full (100 percent) delivery in networks with more than 50 nodes. Both OSP and DSP have high ( $>90$ percent) delivery ratios, while $\mathrm{HHH}$ has a relatively low (79 percent) delivery ratio. That is because all 
other protocols are deterministic and guarantee full delivery in an ideal network, while HHH is a probabilistic scheme and does not guarantee full delivery.

Collision. The signal interference range in TeNs is more realistic and larger than that in $n s 2$. In addition, the current IEEE 802.11 MAC is defected, which cannot avoid collisions among multicast traffics using directional antennas (called the directional hidden terminal problem): When a node $u$ is multicasting to a certain direction, another node $v$ in the opposite direction cannot sense the transmission even when $v$ is close to $u$. In this case, $v$ may transmit to the same direction and cause collisions at many receivers. In the simulation, we use a random forward jitter delay in $\left[0, D_{\max }\right]\left(D_{\max }=100 \mathrm{~ms}\right)$ to reduce collisions, and limit the traffic load to no higher than 10pps.

Fig. $8 \mathrm{~d}$ shows delivery ratios under low mobility $(1 \mathrm{~m} / \mathrm{s})$ and low traffic (1pps). All protocols have lower delivery ratio with a small jitter delay $\left(D_{\max }=1-10 \mathrm{~ms}\right)$. Even Flooding cannot achieve full delivery in this case; the delivery ratio of $\mathrm{HHH}$ is as low as 60 percent. The delivery ratios increase quickly as $D_{\max }$ increases, and reach their maximums when $D_{\max }=100 \mathrm{~ms}$. Such a jitter delay is used by other simulations in this section. However, a large $D_{\max }$ causes high end-to-end delay, as shown in Fig. 8e. A novel collision avoidance mechanism at the MAC layer is crucial for effective broadcast/multicast using directional antennas.

Mobility. Fig. 8f shows the effect of mobility on the delivery ratio. Flooding and SHJ are barely affected by mobility, as they have sufficient redundancy to overcome packet losses in node movement. On the other hand, the delivery ratios of OSP, DSP, and HHH decrease as moving speed increases. DSP has about the same level of redundancy as $\mathrm{HHH}$, but has a much higher delivery ratio than $\mathrm{HHH}$. The reason is that $\mathrm{HHH}$ designates only one neighbor in each direction. No neighbor will forward the packet if this neighbor misses the packet due to node movement.

Simulation results in this section can be summarized as follows:

1. DSP is more efficient than OSP, and much more efficient than SHJ.

2. All broadcast protocols using directional antenna suffer from packet collisions caused by the directional hidden terminal problem. This problem can be alleviated by introducing a jitter delay at each forward node.

3. DSP has nearly the same efficiency as $\mathrm{HHH}$, but is much more reliable than $\mathrm{HHH}$ in realistic networks with collision and mobility.

\section{Conclusion}

We have proposed an efficient broadcast protocol for ad hoc networks using directional antennas. This protocol, called directional self-pruning (DSP), is a nontrivial generalization of an existing localized deterministic broadcast protocol using omnidirectional antennas. Compared with its omnidirectional predecessor, DSP achieves much lower broadcast redundancy and conserves bandwidth and energy consumption. DSP is based on 2-hop topology information and does not rely on any location or angle-of-arrival (AoA) information. A special case of DSP can be used for preserving shortest paths in on-demand route discovery processes. Another special case of DSP is proposed to use the directional reception mode in broadcasting. We proved that the average number of forward nodes in DSP is within a constant factor of the minimal value in an optimal solution. Extensive simulation results show that DSP outperforms many existing directional and omnidirectional broadcast protocols in terms of efficiency and/or reliability.

In future work, we plan to expand the proposed scheme to support neighbor-designating protocols such as MPR and its variations [14], [15], [21], and use a "cleaner" scheme for using the directional reception mode in efficient broadcasting. The current version of DSP-DR relies on a virtual backbone for determining the reception directions of each node. A more elegant approach should treat all nodes uniformly. Another task is the probabilistic analysis on the number of forward directions in random ad hoc networks. One possibility is to extend existing results [16] from onehop networks to multihop networks. We also plan to develop MAC layer support for effective multicast/broadcast using directional antennas via mitigating the directional hidden terminal problem.

\section{ACKNOWLEDGMENTS}

This work was supported in part by US National Science Foundation grants CCR 0329741, CNS 0422762, CNS 0434533, ANI 0073736, and EIA 0130806.

\section{References}

[1] K.M. Alzoubi, P.J. Wan, and O. Frieder, "Distributed Heuristics for Connected Dominating Sets in Wireless Ad Hoc Networks," J. Comm. and Networks, vol. 4, no. 1, pp. 22-29, Mar. 2002.

[2] L. Bao and J.J. Garcia-Luna-Aceves, "Transmission Scheduling in Ad Hoc Networks with Directional Antennas," Proc. ACM MobiCom, pp. 48-58, Sept. 2002.

[3] J. Cartigny and D. Simplot, "Border Node Retransmission Based Probabilistic Broadcast Protocols in Ad-Hoc Networks," Telecomm. Systems, vol. 22, nos. 1-4, pp. 189-204, 2003.

[4] R.R. Choudhury and N.H. Vaidya, "Ad Hoc Routing Using Directional Antennas," technical report, Dept. Electrical and Computer Eng., Univ. of Illinois at Urbana Champaign, May 2002.

[5] R.R. Choudhury, X. Yang, R. Ramanathan, and N.H. Vaidya, "Using Directional Antennas for Medium Access Control in Ad Hoc Networks," Proc. ACM MobiCom, pp. 59-70, Sept. 2002.

[6] F. Dai and J. Wu, "An Extended Localized Algorithm for Connected Dominating Set Formation in Ad Hoc Wireless Networks," IEEE Trans. Parallel and Distributed Systems, vol. 15, no. 10, pp. 902-920, Sept. 2004.

[7] B. Das, R. Sivakumar, and V. Bhargavan, "Routing in Ad Hoc Networks Using a Spine," Proc. IEEE IC3N, pp. 1-20, Sept. 1997.

[8] K. Fall and K. Varadhan, "The NS Manual," The VINT Project, UCB, LBL, USC/ISI, and Xerox PARC, http://www.isi.edu/ nsnam/ns/doc/, Apr. 2002.

[9] C. Hu, Y. Hong, and J. Hou, "On Mitigating the Broadcast Storm Problem with Directional Antennas," Proc. of IEEE ICC, May 2003.

[10] D. Simplot-Ryl, J. Cartigny, and I. Stojmenovic, "An Adaptive Localized Scheme for Energy Efficient Broadcasting in Ad Hoc Networks with Directional Antennas," Proc. Ninth IFIP PWC, pp. 399-413, 2004.

[11] Y.B. Ko, V. Shankarkumar, and N.H. Vaidya, "Medium Access Control Protocols Using Directional Antennas in Ad Hoc Networks," Proc. IEEE Infocom, pp. 13-21, 2000.

[12] P.H. Lehne and M. Pettersen, "An Overview of Smart Antenna Technology for Mobile Communication Systems," IEEE Comm. Surveys, vol. 2, no. 4, pp. 2-13, Nov.-Dec. 1999. 
[13] W.H. Liao, Y.C. Tseng, and J.P. Sheu, "GRID: A Fully LocationAware Routing Protocol for Mobile Ad Hoc Networks," Telecomm. Systems, vol. 18, pp. 37-60, 2001.

[14] H. Lim and C. Kim, "Multicast Tree Construction and Flooding in Wireless Ad Hoc Networks," Proc. ACM MSWiM, Aug. 2000.

[15] W. Lou and J. Wu, "On Reducing Broadcast Redundancy in Ad Hoc Wireless Networks," IEEE Trans. Mobile Computing, vol. 1, no. 2, pp. 111-123, Apr.-June 2002.

[16] K. Nakano, S. Olariu, and A.Y. Zomaya, "Energy-Efficient Routing in the Broadcast Communication Model," IEEE Trans. Parallel and Distributed Systems, vol. 13, no. 12, pp. 1201-1210, Dec. 2002.

[17] A. Nasipuri, S. Ye, J. You, and R.E. Hiromoto, "A MAC Protocol for Mobile Ad Hoc Networks Using Directional Antennas," Proc. IEEE WCNC, Sept. 2000.

[18] W. Navidi and T. Camp, "Stationary Distributions for the Random Waypoint Mobility Model," IEEE Trans. Mobile Computing, vol. 3, no. 1, pp. 99-108, Jan.-Mar. 2004.

[19] S. Olariu, A. Wadaa, L. Wilson, and M. Eltoweissy, "Wireless Sensor Networks: Leveraging the Virtual Infrastructure," IEEE Network, vol. 14, no. 4, pp. 51-56, July/Aug. 2004.

[20] W. Peng and X. Lu, "On the Reduction of Broadcast Redundancy in Mobile Ad Hoc Networks," Proc. ACM MobiHoc, pp. 129-130, Aug. 2000.

[21] A. Qayyum, L. Viennot, and A. Laouiti, "Multipoint Relaying for Flooding Broadcast Message in Mobile Wireless Networks," Proc. HICSS, vol. 9, p. 298, Jan. 2002.

[22] R. Ramanathan, "On the Performance of Ad Hoc Networks with Beamforming Antennas," Proc. ACM MobiHoc, pp. 95-105, Oct. 2001.

[23] M.Q. Rieck, S. Pai, and S. Dhar, "Distributed Routing Algorithms for Multi-Hop Ad Hoc Networks Using D-Hop Connected DDominating Sets," Computer Networks J. (COMNET), vol. 47, no. 6, pp. 785-799, Apr. 2005.

[24] S. Roy and A. Kumar, "Realistic Support for IEEE802. 11b MAC in NS," Bachelor's thesis, Indian Inst. of Technology, Kanpur, May 2004.

[25] C.C. Shen, Z. Huang, and C. Jaikaeo, "Directional Broadcast for Ad Hoc Networks with Percolation Theory," technical report, Computer and Information Sciences, Univ. of Delaware, Feb. 2004.

[26] J. Sucec and I. Marsic, "An Efficient Distributed Network-Wide Broadcast Algorithm for Mobile Ad Hoc Networks," CAIP Technical Report 248, Rutgers Univ., Sept. 2000.

[27] M. Takai, J. Martin, A. Ren, and R. Bagrodia, “Directional Virtual Carrier Sensing for Directional Antennas in Mobile Ad Hoc Networks," Proc. ACM MobiHoc, pp. 183-193, June 2002.

[28] Y.C. Tseng, S.Y. Ni, Y.S. Chen, and J.P. Sheu, "The Broadcast Storm Problem in a Mobile Ad Hoc Network," Wireless Networks, vol. 8, nos. 2-3, pp. 153-167, Mar.-May 2002.

[29] J.E. Wieselthier, G.D. Nguyen, and A. Ephremides, "EnergyAware Wireless Networking with Directional Antennas: The Case of Session-Based Broadcasting and Multicasting," IEEE Trans. Mobile Computing, vol. 1, no. 3, pp. 176-191, July-Sept. 2002.

[30] B. Williams and T. Camp, "Comparison of Broadcasting Techniques for Mobile Ad Hoc Networks," Proc. MobiHoc, pp. 194-205, June 2002.

[31] J. Wu and F. Dai, "Broadcasting in Ad Hoc Networks Based on Self-Pruning," Proc. IEEE Infocom, Mar.-Apr. 2003.

[32] J. Wu and H. Li, "On Calculating Connected Dominating Set for Efficient Routing in Ad Hoc Wireless Networks," Proc. Dial M, pp. 7-14, 1999.

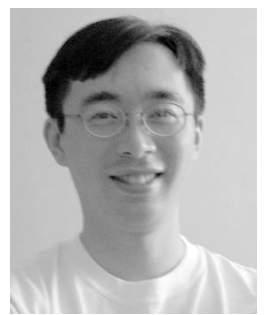

Fei Dai received the $\mathrm{PhD}$ degree from the Department of Computer Science and Engineering at Florida Atlantic University and the MS degree from the Department of Computer Science and Technology at Nanjing University, China. $\mathrm{He}$ is an assistant professor in the Department of Electrical and Computer Engineering at North Dakota State University. He has been a senior programmer at Greatwall Computer and a software architect and team leader at J\&A Securities, both in China. His research interets include networking mobile computing, parallel and distributed computing, artificial intelligence, and software engineering. $\mathrm{He}$ is a member of the IEEE and the IEEE Computer Society.

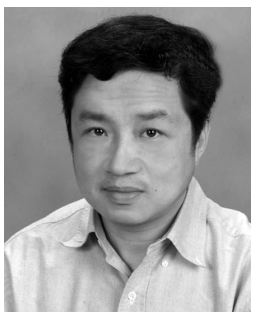

Jie $\mathbf{W u}$ is a professor in the Department of Computer Science and Engineering, Florida Atlantic University. He has published more than 200 papers in various journal and conference proceedings. His research interests are in the area of mobile computing, routing protocols, fault-tolerant computing, and interconnection networks. Dr. Wu served as a program vice chair for the 2000 International Conference on Paralle Processing (ICPP) and a program vice chair for the 2001 IEEE International Conference on Distributed Computing Systems (ICDCS). He is a program cochair for the IEEE First International Conference on Mobile Ad-Hoc and Sensor Systems (MASS '04). He was a coguest editor of a special issue of IEEE Computer on ad hoc networks. He also edited several special issues in Journal Parallel and Distributing Computing (JPDC) and IEEE Transactions on Parallel and Distributed Systems (TPDS). He is the author of the text Distributed System Design (CRC Press). Currently, Dr. Wu serves as an associate editor for IEEE Transactions on Parallel and Distributed Systems and three other international journals. Dr. Wu is a recipient of the 1996-1997 and 2001-2002 Researcher of the Year Award at Florida Atlantic University. He served as an IEEE Computer Society Distinguished Visitor, and is currently the chair of the IEEE Technical Committee on Distributed Processing (TCDP). Dr. Wu is a member of the ACM and a senior member of the IEEE and the IEEE Computer Society.

For more information on this or any other computing topic, please visit our Digital Library at www.computer.org/publications/dlib. 